\title{
Epidemic Update and Risk Assessment of 2019 Novel Coronavirus - China, January 28, 2020
}

\author{
Wenxiao Tu' ${ }^{1}$; Houlin Tang²; Fangfang $\mathrm{Chen}^{2}$; Yinong Wei ${ }^{1}$; Tingling Xü ${ }^{3}$ Kaiju Liao ${ }^{4}$; \\ Nijian Xiang'; Guoqing Shi'; Qun Li ${ }^{1}$; Zijian Feng ${ }^{1, *}$
}

\section{Background}

On December 29, 2019, the health authorities in Hubei Province and Wuhan City received a report of a cluster of pneumonia cases of unknown etiology from a local hospital, and all four patients were employees of the Wuhan Huanan Seafood Wholesale Market (HN market for short) (1). By January 8, 2020, Chinese authorities had identified a new virus 2019 novel coronavirus (2019-nCoV) linked with the outbreak of disease, which has been named novel coronavirusinfected pneumonia (NCIP) (2). On 15 January, the National Health Commission (NHC) issued guidelines for the diagnosis, treatment, prevention, and control of NCIP. These guidelines directed case monitoring, reporting, diagnosis, treatment, management, close contact management, and laboratory testing. On
January 20, the State Council agreed to include NCIP into the Management of the Infectious Diseases Law and the Health and Quarantine Law. On January 25, the Central Committee of the Communist Party of China set up a leading group to deal with this epidemic.

\section{Epidemic Status}

The latest case numbers can be seen on China CDC website: http://114.116.95.250/2019-nCoV/index. html. The geographic distribution and epidemic curve as of January 27, 2020 are shown in Figures 1 and 2.

NCIP in Wuhan continues to spread and the number of cases is still on the rise. The virus has spread to 11 prefectures of Hubei Province outside Wuhan, including Huanggang, Xiaogan, Jingmen, and others.

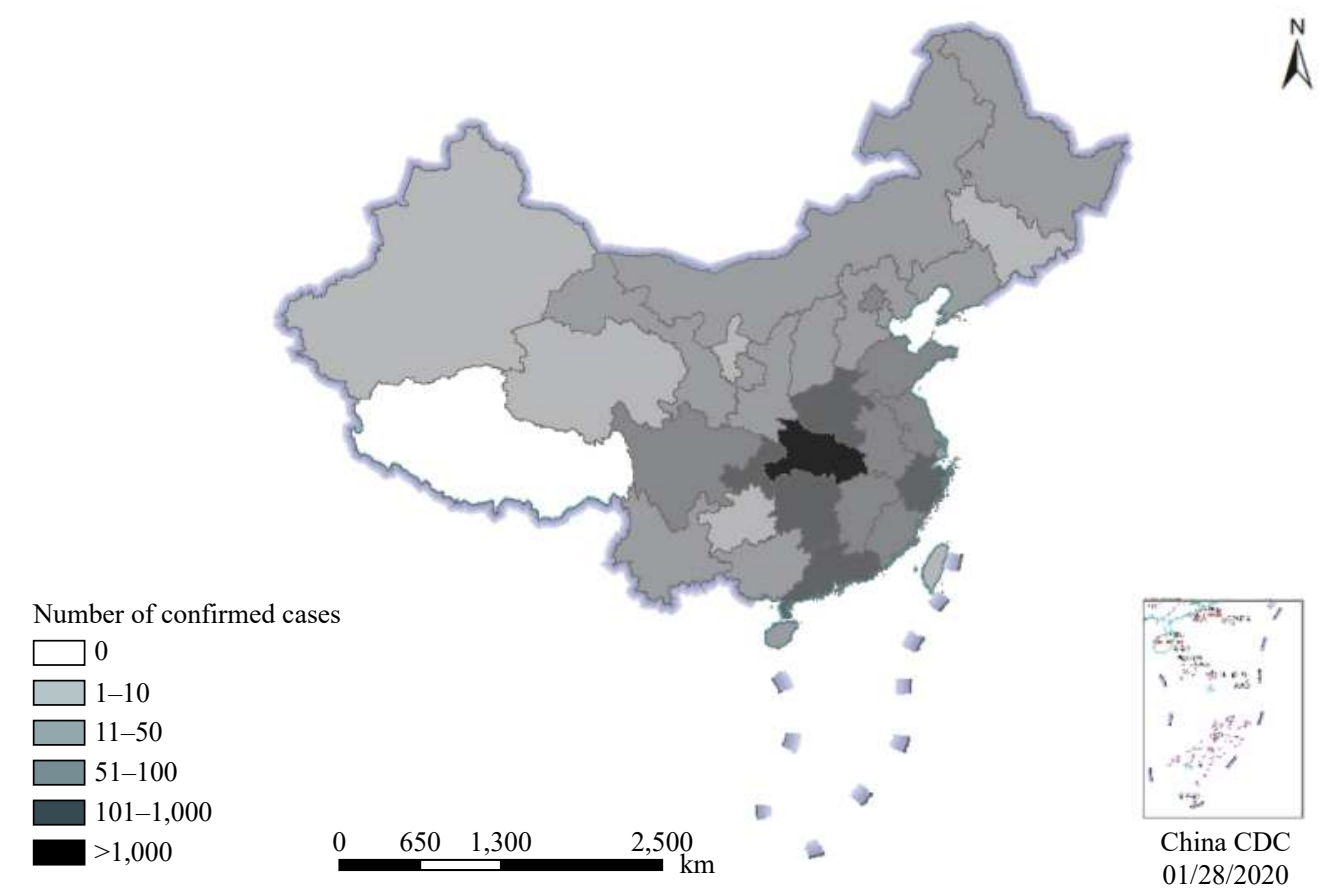

FIGURE 1. The distribution of confirmed cases of 2019 novel coronavirus (2019-nCoV) in China. (as of 24:00 January 27, 2020) 


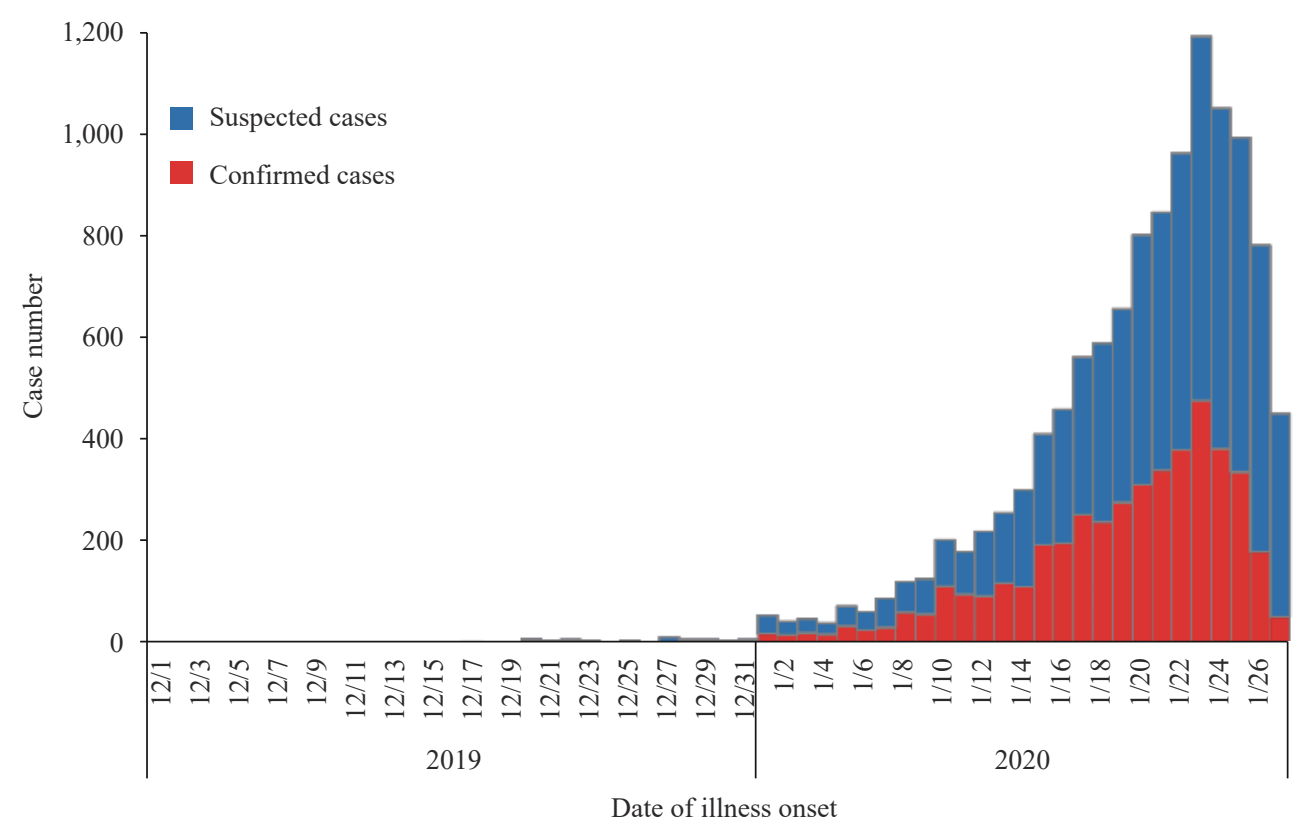

FIGURE 2. Epidemic curve of 2019 novel coronavirus (2019-nCoV) in Mainland China by day. (as of 24:00 January 27, 2020)

Outside of Hubei Province, 29 provinces in Mainland China have reported cases. Currently, most cases are related to Wuhan and others were caused by patients from Wuhan.

Because of the apparent transmission within communities in Wuhan (3) and some areas in Hubei Province, mild early symptoms of the disease, and existence of mild cases, diagnosing the disease and quarantining patients in time is difficult and may potentially cause accumulation of infection sources in the community and increase the difficulty in controlling the disease. For this reason, the number of cases in Wuhan and a few other prefectures in Hubei Province may continue to increase.

Most cases in provinces outside of Hubei Province are imported from Wuhan. There has been no apparent transmission within communities in most of those provinces.

\section{Current Key Knowledge of 2019-nCoV}

Clinical characteristics of 2019-nCoV can be viewed in the Diagnosis and Treatment Guidelines (4). The current male-to-female ratio of all detected cases is $1.2: 1$. The median age is 48 years, ranging from 9 months to 96 years, with less than $0.6 \%$ of cases under the age of 15 years. The incubation period is approximately 3-7 days. Older patients and patients with underlying diseases are prone to severe illness and death. Nucleic acids from 2019-nCoV have been found in influenza-like illness cases in routine surveillance specimens, and there is some evidence of inapparent infection. The clinical infection spectrums are still under investigation.

The results of the field investigation, laboratory testing, and knowledge of other known coronaviruses indicates that the 2019-nCoV originated in wild animals, even though the host animal(s) are still under investigation. Whether the spillover from animals to humans and the completion of adaptive mutation of the virus occurred in $\mathrm{HN}$ market remains to be confirmed, HN market has been identified as the main source of infection for early cases of this epidemic. Current evidence suggests that the main routes of transmission of the virus are through respiratory droplets and close contact. The virus is highly contagious, and its basic reproduction number, i.e. the number cases one infection is suspected to generate, is believed to be between two and three. To date, there is no reliable evidence from field investigations that the disease is contagious during the incubation period.

\section{Public Health Measures}

When the preliminary investigation indicated that the epidemic was spreading around the HN market, the market was immediately closed. At the same time, all cases were isolated and treated and close contacts tracked and followed-up with. Environmental samples from the HN market were collected and tested, and 
case finding, contact tracing, and investigations into the sources of infection were initiated.

Since January 15, suspected cases with travel history to Wuhan were monitored in other regions of China to detect imported cases as early as possible and prevent local transmission. In Wuhan, case surveillance and close contact tracing and management were further strengthened.

Recognizing that $95 \%$ of cases detected outside Wuhan were infected during their stay in Wuhan, Wuhan's city buses, subways, ferries, and long-distance passenger transportation were suspended starting on January 23. Citizens of Wuhan were advised not to leave Wuhan without a special reason. Airports and railway stations' departure channels from Wuhan were temporarily closed. All these measures were taken to reduce the bidirectional flow of people. Since January 23, the other 12 prefecture-level cities in Hubei Province have also suspended public transport, stopped activities involving mass gatherings, strengthened community prevention and control, actively detected and diagnosed patients with fever, and strengthened patient admission.

Since January 20, the Chinese Government has launched joint prevention and control mechanisms among 33 central governmental departments, and a series of measures have been taken, including: very frequent media communication and public communication to raise awareness of disease prevention, stopping mass gatherings, encouraging people to reduce personal gatherings and wear face masks to reduce the chance of infection. Cellphone GPS back tracking technology, travelling and visiting history investigations and other measurements have been taken to improve the early detection of cases and reduce spreading.

As of January 25, 30 provinces in China with confirmed cases have launched the highest public health emergency response. China has made great efforts to the community mobilization, which mainly depends on sub-district/township and community/ village cadres, primary health care centers and family/village doctors. At the same time, residents and volunteers are encouraged to participate in the full- or part-time working teams engaging in door-to-door health promotion, case detection, and management. Early referral of patients to hospital for medical treatment and management should be ensured to avoid further transmission. Medical doctors and nurses will be mobilized nationwide and deployed to support epidemic center areas, in order to restore normal medical care as soon as possible. The cases will be isolated and treated, and nosocomial infection control will be effectively implemented. In addition, home isolation of mild cases under the guidance of health professionals is encouraged to reduce the burden on the health care system and prevent nosocomial transmission.

\section{Risk Assessment}

Current evidence suggests that NCIP has been spreading in humans for more than a month. The main epidemic areas are concentrated in and around Wuhan, and the epidemic is still in its early stage. Authorities at all levels in China have taken strong response measures by mobilizing social forces to promote case detection and management to avoid further transmission, enhancing infection control to avoid outbreaks of nosocomial infection, and encouraging the public to reduce gatherings, travel, and wear masks and to wash hands frequently to reduce exposure and infection opportunities. In Wuhan, vigorous measures have been taken such as suspension of operations for urban buses, the subway, ferries, and long-distance passenger transports, and temporary closure of airports and railway stations. Other prefectures in Hubei Province around Wuhan have also adopted varying degrees of traffic control to restrict the movement and gathering of people. The Chinese Government and people have taken strong and efficient measures to contain this NCIP epidemic, and a larger epidemic, or even pandemic, can be prevented.

\section{Acknowledgements}

This work was supported by National Science and Technology Major Project of China (2018ZX1010 1002-003, 2018ZX10201-002-008-002), National Key Research and Development Project (2018YFC 0809903-02) and the National Natural Science Foundation (NSFC, 71934002).

We thank Dr Chin-Kei Lee, China-WHO Country Office, Dr Anna A. Drexler and Dr Karen Nahapetyan, WHO Regional Office for the Western Pacific, for their valuable opinions about English wording in this note.

\# Corresponding author: Zijian Feng, fengzj@chinacdc.cn.

Public Health Emergency Center, Chinese Center for Disease Control and Prevention (China CDC), Beijing, China; ${ }^{2}$ National 
Center for AIDS/STD Control and Prevention, China CDC, Beijing, China; ${ }^{3}$ National Center for Chronic and Noncommunicable Disease Control and Prevention, Chinese Center for Disease Control and Prevention, Beijing, China; ${ }^{4}$ Peking University Health Science Center, Beijing, China.

Submitted: January 29, 2020; Accepted: February 01, 2020

\section{References}

1. The 2019-nCoV Outbreak Joint Field Epidemiology Investigation Team, Li Q. An outbreak of NCIP (2019-nCoV) infection in China -
Wuhan, Hubei Province, 2019-2020. China CDC Weekly 2020;2(5): 79 - 80. http://weekly.chinacdc.cn/en/article/ccdcw/2020/5/79.

2. Tan WJ, Zhao X, Ma XJ, Wang WL, Niu PH, Xu WB, et al. A novel coronavirus genome identified in a cluster of pneumonia Cases Wuhan, China 2019-2020. China CDC Weekly 2020;2(4):61-2. http://weekly.chinacdc.cn/en/article/ccdcw/2020/4/61.

3. Li Q, Guan XH, Wu P, Wang XY, Zhou L, Tong YQ, et al. Early transmission dynamics in Wuhan, China, of novel coronavirus-infected pneumonia. N Engl J Med 2020. http://dx.doi.org/10.1056/NEJMoa 2001316.

4. National Health Commission. Diagnosis and treatment guideline on pneumonia infection with 2019 novel coronavirus (the fourth trial edition). http://www.nhc.gov.cn/yzygj/s7653p/202001/4294563ed35b43 209b31739bd0785e67.shtml. [2020-1-28]. (In Chinese). 